\title{
Glaucomatous Damage Secondary to Long Term Topical Corticosteroid Use in a 7-Year-Old Female
}

\author{
Michael Greenwood, Florin Grigorian \\ Department of Ophthalmology, University Hospitals Case Medical Center, Cleveland, USA \\ Email: Michael.greenwood@UHhospitals.org
}

Received 8 January 2014; revised 5 February 2014; accepted 13 February 2014

Copyright $(02014$ by authors and Scientific Research Publishing Inc.

This work is licensed under the Creative Commons Attribution International License (CC BY). http://creativecommons.org/licenses/by/4.0/

(c) (i) Open Access

\begin{abstract}
Introduction: Topical corticosteroids are commonly prescribed for children with atopic dermatitis. Although generally well tolerated, long term unsupervised use can lead to ocular damage, including glaucoma. Much of the current literature focuses on the periorbital use in older patients. We present a case of an adolescent who suffered glaucomatous damage secondary to long term topical steroid use on her extremities. Methods: This is a case report of a child with extensive work-up for glaucoma suspect. Results: A 7-year-old African American female was found to have glaucomatous damage after using $2.5 \%$ hydrocortisone cream on her arms, legs, and back of neck twice daily continuously for 2 years. There were no other mechanisms that could have produced a secondary glaucoma. Congenital anomalous disk is a remote possibility. Discussion: Steroid cream use for atopic dermatitis is relatively common. The side effect profile of topical corticosteroids has been well documented and includes glaucoma. These patients may not have any visual symptoms, and can present at an advanced stage, with irreversible vision loss. Children with atopic dermatitis are especially susceptible to the systemic effects of steroids because of the damaged epithelial layer and also the body surface area to volume, both of which increase the bioavailability of the medication. Conclusion: Because topical steroids are commonly used, especially in children, patients need to be aware of the potentially dangerous side effects, including vision loss from glaucoma.
\end{abstract}

\section{Keywords}

Glaucoma; Corticosteroids; Atopic Dermatitis

\section{Introduction}

There are several different drugs that have the potential to cause the elevation of intraocular pressure (IOP),

How to cite this paper: Greenwood, M. and Grigorian, F. (2014) Glaucomatous Damage Secondary to Long Term Topical Corticosteroid Use in a 7-Year-Old Female. Open Journal of Pediatrics, 4, 62-66.

http://dx.doi.org/10.4236/ojped.2014.41008 
which can occur via an open-angle or a closed-angle mechanism. Steroid-induced glaucoma is a form of openangle glaucoma that is usually associated with topical (ocular) steroid use, but may develop with inhaled, oral, intravenous, intravitreal, or topical (dermatologic) administration. The exact pathophysiology of steroid-induced glaucoma is unknown, but we do know that steroid-induced IOP elevation is secondary to increased resistance to aqueous outflow [1].

Patients with atopic dermatitis are commonly prescribed topical corticosteroids as a primary treatment. Although they are usually well tolerated, they can have adverse effects. Much of the current literature regarding glaucoma and topical dermatologic application focuses on periorbital use in older patients [2]-[5]. These patients may not have any visual symptoms, and can present at an advanced stage, with irreversible vision loss. Children with atopic dermatitis are especially susceptible to the systemic effects of steroids because of the damaged epithelial layer and also the body surface area to volume, both of which increase the bioavailability of the medication [6].

We report a case of glaucomatous damage in a young girl secondary to the prolonged use of topical corticosteroids, a rare but damaging side-effect.

\section{Case Presentation}

A 7-year-old female presented to our clinic after being referred for large optic nerves. She stated that she has had pain in her eyes for the past 2 months, left eye worse than right. She has also had headaches associated with the eye pain. The symptoms have been intermittent, but occurring more frequently. Because of these symptoms, she was taken to an optometrist for possible correction of a refractive error. The abnormal looking nerves were dis- covered, and she was referred to the pediatric ophthalmology clinic. Her past medical history is significant for asthma and atopic dermatitis. She uses an albuterol inhaler as needed, and has been applying hydrocortisone cream (2.5\%) to her arms, legs, and back of her neck twice daily, every day, for the past two years. Her birth and development are unremarkable. Family history is significant for a grandfather and uncle who have glaucoma. Our exam revealed a visual acuity of 20/20- and 20/20 in the right and left eyes respectively. Her stereopsis was within the normal limits for her age group, and her color plates were full in both eyes. Cycloplegic refraction was +0.75 sphere in both eyes, normal for her age group. Pupils, extraocular movements, and alignment were all within normal limits, including no evidence of an afferent pupillary defect. Measurements of IOP were 27 in the right eye, 24 in the left eye via iCare tonometer. External and slit lamp exams were within normal limits. Her dilated fundus exam revealed enlarged cup to disc ratios ( 0.8 right, 0.75 left), undermined margins, mild pallor, bayoneting of vessels, and deep cupping (Figure 1). Gonioscopy revealed open angles 360 degrees to the ciliary body with no iris processes and no hyperpigmentation. Pachymetry testing measured the central corneal thickness (CCT) to be 615 OD, 600 OS. Additional testing was performed over the next few visits to help guide the diagnosis (Figures 2, 3) which included optical coherence tomography (OCT) of the optic nerve head, and Humphrey visual field tests $\times 3$ in order to get a reliable test. Given the headache, pallor of the optic nerves, and
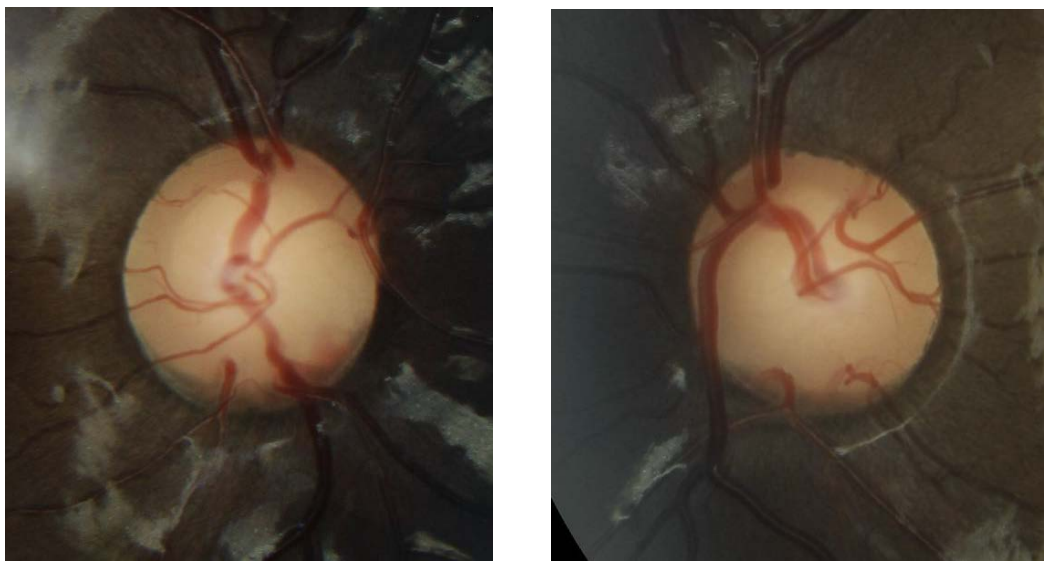

Figure 1. The right and left optic nerves showing enlarged cup to disc ratios (0.8 right, 0.75 left), undermined margins, mild pallor, bayoneting of vessels, and deep cupping. 

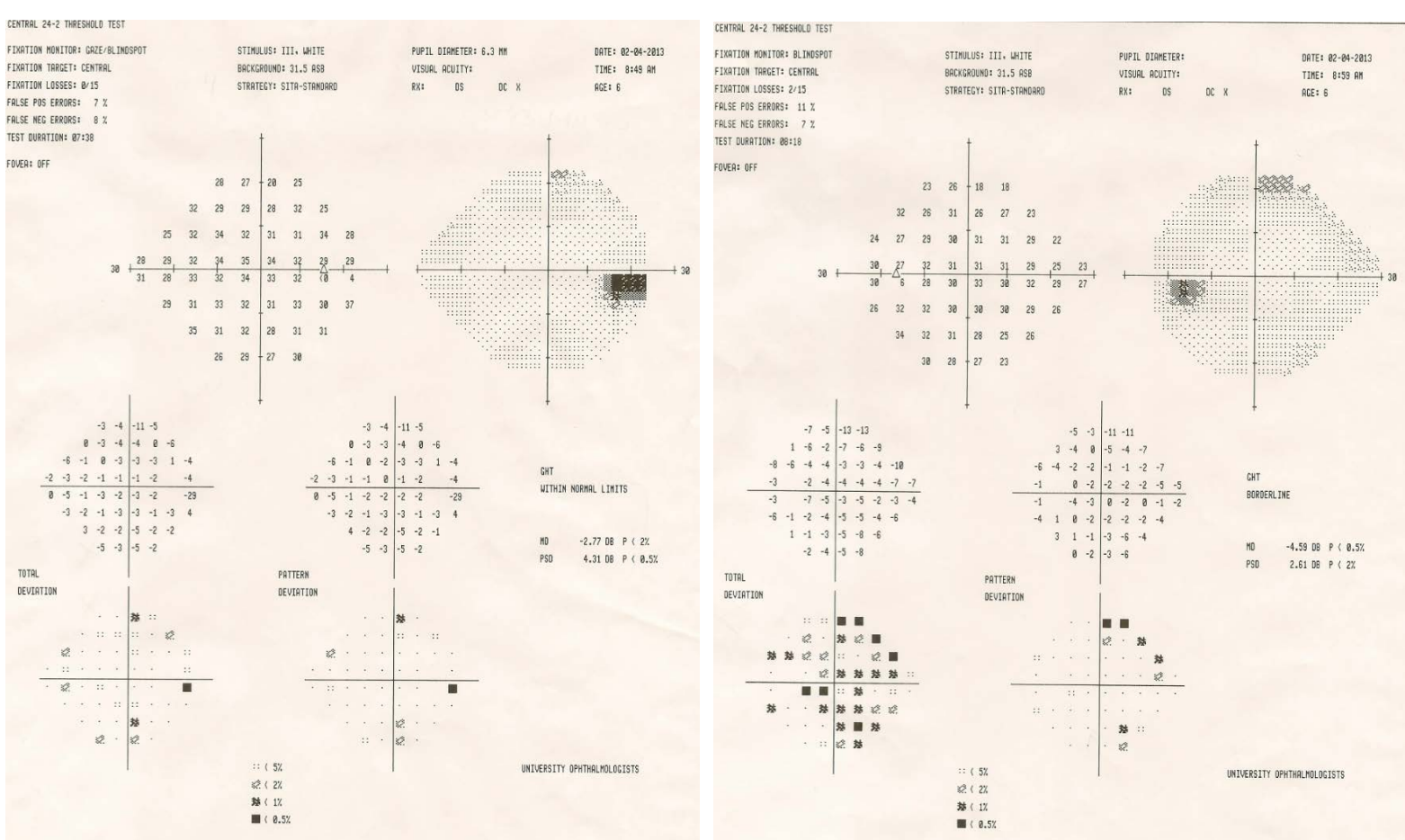

Figure 2. visual field testing showing arcuate defect in the left eye. This is the patient's third visual field test.
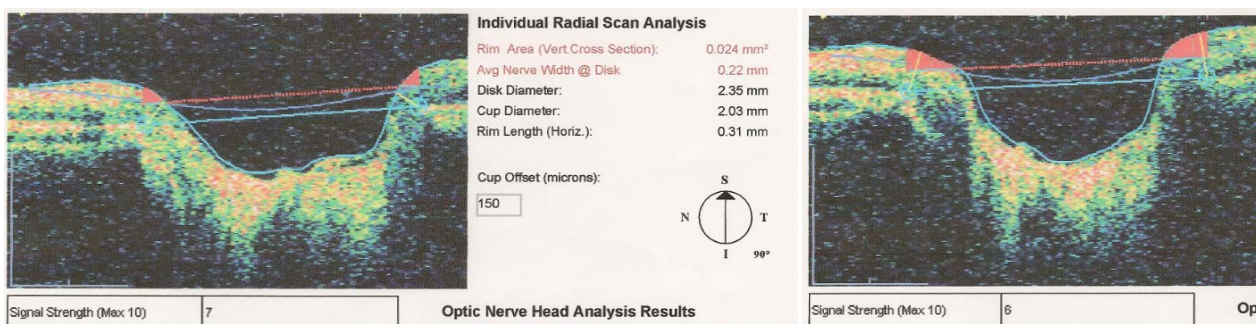

dividual Radial Scan Analysis $\begin{array}{lll}\text { Rim Area (Vort Cross Soction): } & 0.09 \mathrm{~mm}^{2} \\ \text { Avg Nerve Wodth C Disk } & 0.31 \mathrm{~mm}\end{array}$ $\begin{array}{lrl}2.6 \mathrm{~mm} \\ \text { Disk Diameter: } & 1.81 \mathrm{~mm} \\ \text { Cup Diameter: } & 0.70 \mathrm{~mm}\end{array}$
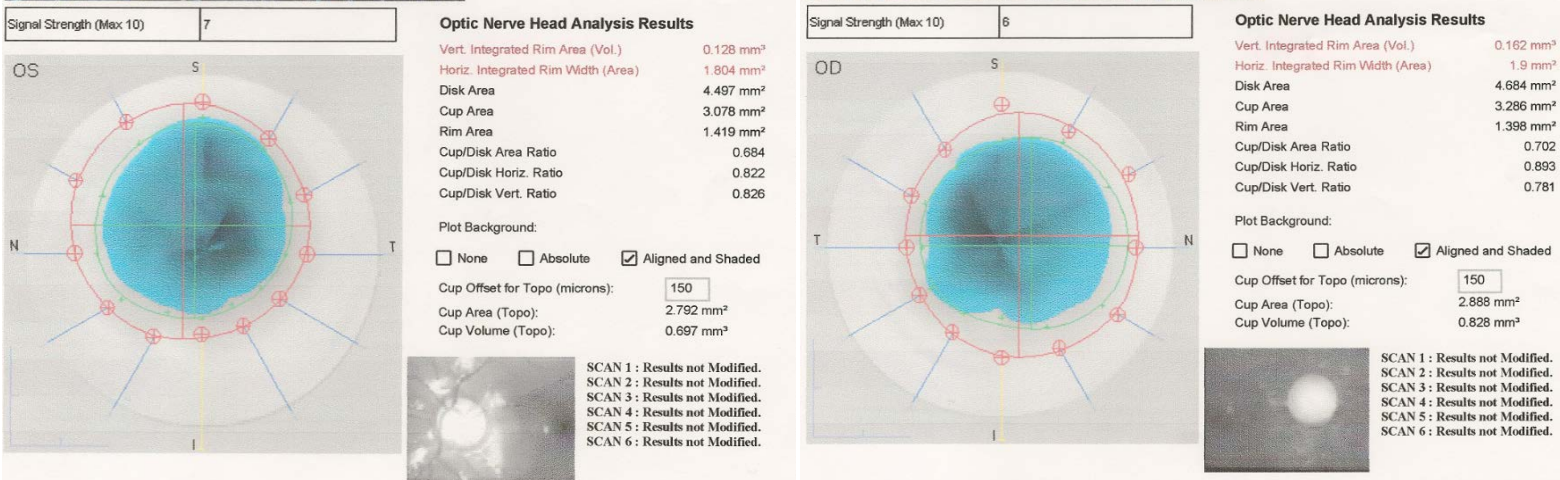

Figure 3. OCT images of the right and left optic nerves. Note the enlarged Cup/Disk ratio and also the enlarged nerve size.

preserved visual field relative to aspect of cups, an alternative etiology for optic neuropathy was considered. For this reason, an MRI of the brain and orbits was performed, which showed normal anatomy with no mass lesions. After the initial visit, she was started on dorzolamide/timolol (Cosopt) twice daily OU and latanoprost (Xalatan) once a day at night OU. She was also instructed to stop the hydrocortisone cream. At the follow up visit one week later, her headaches had decreased somewhat, but she still had eye pain occasionally. Her IOPs were now 17 OD, 22 OS via iCare, and confirmed with applanation tonometry. At her 1 month follow up, the headaches had resolved along with the eye pain. Measurements of IOP were 21 OD, 17 OS via iCare, and 19 OD 17 
OS via applanation.

\section{Discussion}

Topical corticosteroids are commonly used treat atopic dermatitis in the pediatric population. They are usually dosed as a low- or medium-strength steroid used once or twice daily for a short period of time followed by a period of no application of equal length. Lower-potency agents are preferred in infants and children because of the increased surface-to-weight ratio. It is also important to consider the location that the corticosteroid is applied, as this can affect absorption. The forearm has poor absorption, but neck and face absorb increased amounts and a more likely to develop local side effects. This is believed to be a result of the thickness of the statum corneum. The defective epidermal barrier that is present in patients with atopic dermatitis allows for 2 to 10 times greater penetration than in healthy skin [6].

The formation of glaucoma from the use of topical corticosteroids around the eye is a controversial topic in the literature. Although there are no formal studies regarding topical corticosteroids and the formation of glaucoma, many case reports exist. Early reports by Cubey [2], Zugerman [3], Neilsen [4], and Sahni [5] support this finding, while Haeck [7] found that frequent application of corticosteroids to the eyelids in patients with atopic dermatitis did not develop glaucoma or cataracts, another common problem associated with corticosteroid use. Most of the above reports only take into consideration the use of steroids around the eyelids and orbit. Our case is unique in that the medication was used extensively on the extremities and neck for a prolonged period of time with no interval dosing.

Although the exact pathophysiology of steroid-induced glaucoma is currently not known, it is known that steroid-induced IOP elevation is secondary to increased resistance to aqueous outflow. What is important to keep in mind is that these patients are often unaware of the damage it may be causing because many of them are asymptomatic, and by the time they present, their visual impairment may be advanced. This is compounded by the fact that the patient usually is seeing improvement with their dermatologic condition, making it difficult to alter their current treatment.

Patients who require long-term corticosteroid use should be monitored closely for any side-effects and should be warned of potential side-effects. It is essential that they take the medications as prescribed and allow for a period of drug-free time. This is especially important for use around the eyes.

Our case shows evidence of glaucomatous damage secondary to long term corticosteroids in a young patient with atopic dermatitis. She has several risk factors for developing systemic complications secondary to corticosteroid use. These include long-term, uninterrupted use, large surface-to-weight ratio, damaged skin barrier secondary to atopy, and a family history of glaucoma. It is unclear at this point how much glaucomatous damage has been done. This is confounded by the fact that our patient does have some features of an anomalous disc, most notably the size, which makes identifying the abnormalities that could be due to the glaucoma more difficult. Nonetheless, the concern for glaucoma is valid and it would be unsafe to discontinue with the medications. The typical course is for the IOP to return to normal following removal of the corticosteroid, but given the long duration of application, this may not occur. This patient should be followed over the long-term for IOP measurements and continued visual field analysis.

\section{Conclusions}

Topical corticosteroid use is an effective treatment for atopic dermatitis. It is often well tolerated, but the negative side-effect profile must be kept in mind when prescribing these medications. Long-term use can result in irreversible glaucomatous damage if abuse occurs.

Our case demonstrates an unusual presentation as the patient did not use the medication in a periorbital area, but rather in her extremities and neck.

\section{References}

[1] Rhee, D. J., Roy, H. and Gedde, S. (2012) Drug-Induced Glaucoma. Medscape.

[2] Cubey, R.B. (1976) Glaucoma Following Application of Corticosteroid to the Skin of the Eyelids. British Journal of Dermatology, 95, 207-208. http://dx.doi.org/10.1111/j.1365-2133.1976.tb00830.x

[3] Zugerman, C., Sauders, D. and Levit, F. (1976) Glaucoma from Topically Applied Steroids. Archives of Dermatology, 
112, 1326. http://dx.doi.org/10.1001/archderm.1976.01630330080032

[4] Neilsen, N.V. (1978) Sorenson PN. Glaucoma Induced by Application of Corticosteroids to the Periorbital Region. Archives of Dermatology, 114, 953-954. http://dx.doi.org/10.1001/archderm.1978.01640180085023

[5] Sahni, D. (2004) Glaucoma Induced by Periorbital Topical Steroid Use-A Rare Complication. Clinical and Experimental Dermatology, 29, 617-619. http://dx.doi.org/10.1111/j.1365-2230.2004.01610.x

[6] Hengge, U.R., Ruzicka, T., Schwartz, R.A. and Cork, M.J. (2006) Adverse Effects of Topical Glucocorticosteroids. Journal of the American Academy of Dermatology, 54, 16-18. http://dx.doi.org/10.1016/j.jaad.2005.01.010

[7] Haeck, I.M., Rouwen, T.J., Timmer-de Mik, L., de Bruin-Weller, M.S. and Bruijnzeel-Koomen, C.A. (2011) Topical Corticosteroids in Atopic Dermatitis and the Risk of Glaucoma and Cataracts. Journal of the American Academy of Dermatology, 64, 275-281. http://dx.doi.org/10.1016/j.jaad.2010.01.035 